\title{
Assessment of models and methods for pressurized spherical composites
}

\section{David Guinovart-Sanjuán}

Facultad de Matemática y Computación, Universidad de La Habana, Cuba; Department of Mathematics, University of Central Florida, USA

\section{Raffaella Rizzoni}

Dipartimento di Ingegneria, Università di Ferrara, Italy

\section{Reinaldo Rodríguez-Ramos}

Facultad de Matemática y Computación, Universidad de La Habana, Cuba

\section{Raúl Guinovart-Díaz}

Facultad de Matemática y Computación, Universidad de La Habana, Cuba

\section{Julián Bravo-Castillero}

Facultad de Matemática y Computación, Universidad de La Habana, Cuba

\section{Ransés Alfonso-Rodríguez}

Facultad de Matemática y Computación, Universidad de La Habana, Cuba; Department of Mathematics, University of Central Florida, USA

\section{Frederic Lebon}

Laboratoire de Mécanique et d'Acoustique, Aix-Marseille University, France; Aix-Marseille University, CNRS, Centrale Marseille, LMA, France

\section{Serge Dumont}

University of Nîmes, France

\section{Federico J Sabina}

Instituto de Investigaciones en Matemáticas Aplicadas y en Sistemas,

Universidad Nacional Autónoma de México, México. 


\begin{abstract}
The elastic properties of a spherical heterogeneous structure with isotropic periodic components is analyzed and a methodology is developed using the two-scale asymptotic homogenization method (AHM) and spherical assemblage model (SAM). The effective coefficients are obtained via AHM for two different composites: (a) composite with perfect contact between two layers distributed periodically along the radial axis; and (b) considering a thin elastic interphase between the layers (intermediate layer) distributed periodically along the radial axis under perfect contact. As a result, the derived overall properties via AHM for homogeneous spherical structure have transversely isotropic behavior. Consequently, the homogenized problem is solved. Using SAM, the analytical exact solutions for appropriate boundary value problems are provided for different number of layers for the cases (a) and (b) in the spherical composite. The numerical results for the displacements, radial and circumferential stresses for both methods are compared considering a spherical composite material loaded by an inside pressure with the two cases of contact conditions between the layers (a) and (b).
\end{abstract}

\title{
Keywords
}

Spherical composites, mechanical properties, elasticity, analytical modeling, numerical analysis

\section{Introduction}

The mathematical modeling of composite materials is necessary to develop new types of structure with improved characteristics such as strength, lightness and durability. Multi-layered shells are the most popular composite structures [1,2]. They have an important role in engineering, aeronautics and automotive, being lightweight structures resulting from the need to reduce fuel consumption and exhaust emissions [3, 4]. Another important application for shell composites is in the textile industry. Textile composite materials have recently received considerable attention, due to their structural advantages of high specific strength and high specific stiffness as well as their improved resistance to impact [5,6]. Textile composites have an important application in sports, where the development of new materials via physical and mathematical design has changed the usual perspective and strategy. For example, the development of new, resistant, aerodynamic and elastic balls has improved the attractiveness of sports such as soccer [7] and baseball [8,9].

It is well known that the presence of interfaces plays a crucial role in the behavior of composite structures and in recent years there has been considerable interest in the study of composites with imperfect interfaces $[10,11]$.

In this work, the elastic properties of a spherical laminated shell composite are studied together with the influence of the stress and strain distributions for two different composites: (a) composite with perfect contact between two layers distributed periodically along the radial axis; and (b) considering a thin elastic interphase (intermediate layer) between the layers distributed periodically along the radial axis under perfect contact $[12,13]$. The two-scale asymptotic homogenization method (AHM) is a powerful tool to obtain the effective elastic properties of laminated shell composites, being suitable to take into account the above-mentioned cases (a) and (b) [14-16]. The expressions of the effective coefficients for a laminated shell composite considered in the cases (a) and (b) using AHM are obtained. As a consequence of the homogenization process the derived overall properties for homogeneous spherical structure have transversely isotropic behavior $[17,18]$. This symmetry class provides an excellent framework for the constitutive model development of many types of composites, including soft tissues [19, 20]. The general solution of the homogenized equilibrium equation for a transversely isotropic structure is obtained and the expression of the stress tensor is computed. Besides, the analytical exact solutions for appropriate boundary value problems are provided using the spherical assemblage model (SAM), reported in [13], but now extended to different number of layers for the cases (a) and (b) in the spherical composite. The solution of the elastic problem for a spherical structure is presented for the two cases (a) and (b). In particular, the solutions for the displacements, radial and angular stresses fields obtained by AHM and SAM are compared for different numbers of layers.

\section{Statement of the heterogeneous elastic problem}

A laminated shell composite $\Omega \subset \mathbb{R}^{3}$ is considered with infinite number of layers periodically distributed along a preferential direction $[21,22]$, bounded by the surfaces $\Gamma_{1} \Gamma_{2}, \Gamma_{1} \cap \Gamma_{2}=\emptyset$, where the axis $x_{3}$ is perpendicular to 
the layers of the media. The three-dimensional equilibrium problem on an elastic body is given by the equations

$$
\begin{gathered}
\operatorname{div} \boldsymbol{\sigma}+\boldsymbol{f}=\mathbf{0}, \quad \text { on } \Omega, \\
\boldsymbol{u}=\boldsymbol{u}^{0} \quad \text { on } \Gamma_{1}, \quad \boldsymbol{\sigma} \cdot \mathbf{n}=\mathbf{S}^{0} \quad \text { on } \Gamma_{2}, \\
\llbracket \boldsymbol{u} \rrbracket=0 \quad \text { on } \Gamma, \\
\llbracket \boldsymbol{\sigma} \cdot \mathbf{n} \rrbracket=0 \quad \text { on } \Gamma,
\end{gathered}
$$

where $\boldsymbol{\sigma}$ is the stress tensor, $\boldsymbol{f}$ is a body force density vector acting on $\Omega, \boldsymbol{u}$ is the displacement vector, $\mathbf{n}$ is the normal vector of $\Gamma_{2}, \boldsymbol{u}^{0}$ and $\mathbf{S}^{0}$ are the given displacement on $\Gamma_{1}$ and surface load on $\Gamma_{2}$, respectively, $\llbracket \bullet \rrbracket=\bullet(1)-\bullet(2)$ denotes the difference of the function values at the interface $\Gamma$ between the components of the composite $[12,16,23,24]$.

The problem (1)-(2) can be reformulated in terms of the displacement vector $\boldsymbol{u}$ using the Hooke's law which relates the stress-strain and the Cauchy's formula

$$
\begin{gathered}
\operatorname{div}(\boldsymbol{C}: \nabla \boldsymbol{u})+\boldsymbol{f}=\mathbf{0}, \quad \text { on } \Omega, \\
\boldsymbol{u}=\boldsymbol{u}^{0} \quad \text { on } \Gamma_{1}, \quad(\boldsymbol{C}: \nabla \boldsymbol{u}) \mathbf{n}=\mathbf{S}^{0} \quad \text { on } \Gamma_{2} . \\
\llbracket \boldsymbol{u} \rrbracket=0 \quad \text { on } \Gamma, \\
\llbracket(\boldsymbol{C}: \nabla \boldsymbol{u}) \cdot \mathbf{n} \rrbracket=0 \quad \text { on } \Gamma,
\end{gathered}
$$

where ":" denotes the scalar-tensor product and $\nabla$ represent the covariant derivative operator.

In order to obtain equivalent equations with non-oscillating coefficients, the two-scale AHM is used $[21,25,26]$.

\section{I. The two-scale AHM}

The asymptotic expansion for a curvilinear coordinates system is

$$
\boldsymbol{u}=\boldsymbol{v}+\alpha \boldsymbol{N}_{1} \nabla \boldsymbol{v}+\alpha^{2} \boldsymbol{N}_{2} \nabla^{2} \boldsymbol{v} \ldots
$$

where $\alpha=l / L$ is a small parameter associated with rapid fluctuations in the microstructure or local property, where $l$ is the microscopic scale associated with the heterogeneities and $L$ is the macroscopic scale characterizing the extent of the structure, $\boldsymbol{v} \equiv \boldsymbol{v}(\mathbf{x})$ is a continuous function and $N_{i}=N_{i}(\mathbf{y})$ are periodic functions for the variable $\mathbf{y}=\mathbf{x} / \alpha \in \mathbf{Y}$, where $\mathbf{Y}$ is the periodic or unit cell. For the perfect contact model between the layers $\left\langle\boldsymbol{N}_{i}\right\rangle=0$ (see $\left.[21,22,25,26]\right)$.

Substituting the expansion (9) into the equations (5), (6), (8) and solving a set of problems, the fourth-order tensor of the effective coefficients, $\boldsymbol{h}$, can be determined [21, 22, 25, 27]

$$
\boldsymbol{h}=\left\langle\boldsymbol{C}+\frac{\partial}{\partial \mathbf{y}} \boldsymbol{N}_{1}: \boldsymbol{C}\right\rangle,
$$

where $\langle\bullet\rangle$ is the average operator given by the expression

$$
\langle\bullet\rangle=\frac{1}{V_{\mathbf{Y}}} \int_{\mathbf{Y}} \bullet d y,
$$

where $V_{\mathbf{Y}}$ is the volume of $\mathbf{Y}$. The average is affected by the thickness and the distribution of the layer along the unit cell Y. Therefore, the effective coefficients are different depending on the thickness of the materials. The components of the effective coefficients (10) are given by the expression

$$
h_{i j k l}=\left\langle C_{i j k l}+C_{i j m 3} \frac{\partial N_{k l m}}{\partial y_{3}}\right\rangle \text {, }
$$


and the local function $N_{1}$ is obtained solving the local problem

$$
\frac{\partial}{\partial \mathbf{y}}\left(\boldsymbol{C}+\frac{\partial}{\partial \mathbf{y}} \boldsymbol{N}_{1}: \boldsymbol{C}\right)=\mathbf{0}, \quad \text { on } \mathbf{Y} .
$$

For a curvilinear laminated shell composite, where the layers are periodical distributed perpendicular to the $x_{3}$ axis, the local problem (12) in terms of components is given by the equation

$$
\frac{\partial}{\partial y_{3}}\left(C_{i 3 k l}+C_{i 3 m 3} \frac{\partial N_{k l m}}{\partial y_{3}}\right)=0, \quad \text { on } \mathbf{Y} .
$$

The coefficients of the local problem are different depending on the number of materials and the thickness of each layer.

\subsection{Composite with isotropic layers with perfect contact}

Considering a laminated shell composite with isotropic layers distributed perpendicularly to the $x_{3}$ axis with perfect contact at the interface $\Gamma$, i.e. continuity of the displacements and the stresses (7)-(8), then the solution of the local problem (13) is given by the expression

$$
\frac{\partial N_{m n k}}{\partial x_{3}}=C_{m 3 i 3}^{-1}\left\langle C_{i 3 p 3}^{-1}\right\rangle^{-1}\left\langle C_{p 3 q 3}^{-1} C_{q 3 n k}\right\rangle-C_{m 3 i 3}^{-1} C_{i 3 n k} .
$$
$\boldsymbol{h}$ are

Substituting the expression (14) into the equation (11) the non-zero components of the effective coefficients

$$
\begin{aligned}
h_{1111} & =\left\langle C_{1111}\right\rangle+\left\langle C_{1133}\left(C_{3333}\right)^{-1}\right\rangle^{2}\left\langle\left(C_{3333}\right)^{-1}\right\rangle^{-1} \\
& -\left\langle\left(C_{1133}\right)^{2}\left(C_{3333}\right)^{-1}\right\rangle, \\
h_{1122} & =\left\langle C_{1122}\right\rangle+\left\langle C_{1133}\left(C_{3333}\right)^{-1}\right\rangle\left\langle\left(C_{3333}\right)^{-1}\right\rangle^{-1} \\
& \times\left\langle C_{2233}\left(C_{3333}\right)^{-1}\right\rangle-\left\langle C_{1133} C_{2233}\left(C_{3333}\right)^{-1}\right\rangle, \\
h_{1133} & =\left\langle C_{1133}\left(C_{3333}\right)^{-1}\right\rangle\left\langle\left(C_{3333}\right)^{-1}\right\rangle^{-1}, \\
h_{2233} & =\left\langle C_{2233}\left(C_{3333}\right)^{-1}\right\rangle\left\langle\left(C_{3333}\right)^{-1}\right\rangle^{-1}, \\
h_{2222} & =\left\langle C_{2222}\right\rangle+\left\langle C_{2233}\left(C_{3333}\right)^{-1}\right\rangle^{2}\left\langle\left(C_{3333}\right)^{-1}\right\rangle^{-1} \\
& -\left\langle\left(C_{2233}\right)^{2}\left(C_{3333}\right)^{-1}\right\rangle, \\
h_{3333} & =\left\langle\left(C_{3333}\right)^{-1}\right\rangle^{-1} \\
h_{2323} & =\left\langle\left(C_{2323}\right)^{-1}\right\rangle^{-1} h_{1313}=\left\langle\left(C_{1313}\right)^{-1}\right\rangle^{-1}, \\
h_{1212} & =\left\langle C_{1212}\right\rangle .
\end{aligned}
$$

The effective tensor $\boldsymbol{h}$ with average components (15) has a transversely isotropic behavior if the layers have isotropic behavior, therefore, $h_{1111}=h_{2222}, h_{1133}=h_{2233}, h_{2323}=h_{1313}$ and the $h_{1212}=\left(h_{1111}-h_{1122}\right) / 2$, thus, only five coefficients are linearly independent; this result is proved in [21, Chap. 5].

\section{Homogenized equilibrium equation in a spheric shell}

An homogeneous spheric shell made of transversely isotropic material has non-zero stress components

$$
\begin{aligned}
\sigma_{\theta \theta} & =h_{1111} \frac{1}{r} u_{r}+h_{1122} \frac{1}{r} u_{r}+h_{1133} u_{r, r}, \\
\sigma_{\varphi \varphi} & =h_{1122} \frac{1}{r} u_{r}+h_{1111} \frac{1}{r} u_{r}+h_{1133} u_{r, r}, \\
\sigma_{r r} & =h_{1133} \frac{1}{r} u_{r}+h_{2233} \frac{1}{r} u_{r}+h_{3333} u_{r, r},
\end{aligned}
$$




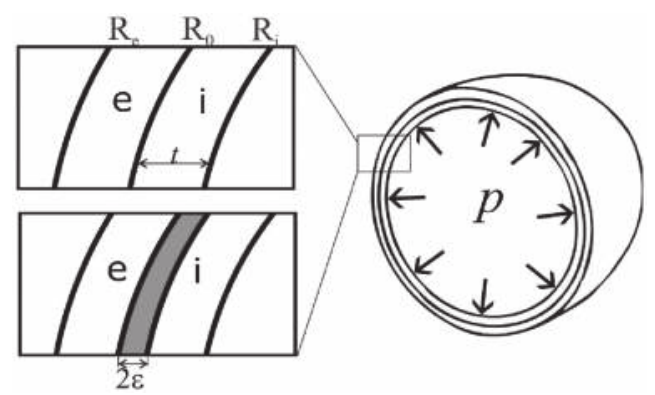

Figure I. Spherical composite element with two layers loaded by a uniform internal pressure.

implying that $\sigma_{\theta \theta}=\sigma_{\varphi \varphi}$.

If the body force vector $\boldsymbol{f}$ in equation (5) is neglected, then the equilibrium equation under axial-symmetric loading conditions is

$$
u_{r, r r}+\frac{2}{r} u_{r, r}+\frac{2\left(h_{1133}-h_{1122}-h_{1111}\right)}{r^{2} h_{3333}} u_{r}=0
$$

whose solution is

$$
u_{r}=c_{1} r^{z_{1}}+c_{2} r^{z_{2}}
$$

with

$$
z_{1,2}=(-1 \pm \sqrt{1-8 B}) / 2,
$$

$B=\left(h_{1133}-h_{1122}-h_{1111}\right) / h_{3333}$ and $c_{1}, c_{2}$ real numbers. For an homogeneous spheric shell made of materials with an isotropic behavior the values of $z_{1,2}$ are 1 and -2 .

\section{Pressurized SAM}

A spherical composite element made of two concentric shells is now considered. The internal shell has inner radius $R_{i}$ and thickness $t$ and the external shell has outer radius $R_{e}$ and same thickness $t$. The inner surface $r=R_{i}$ of the heterogeneous body is loaded by an internal pressure $p$, thus

$$
\sigma_{r r}=-p,
$$

whereas the external spherical surface $r=R_{e}$ is free of traction, see Figure 1 .

In the following, the subscript $i$ denotes quantities associated with the internal shell and the subscript $e$ quantities associated with the external shell. The materials of the two shells are assumed to be linearly elastic, the elastic moduli of the internal shell are $\mu_{i}, \kappa_{i}$ and those of the external spherical shell are $\mu_{e}, \kappa_{e}$.

The three-dimensional homogeneous, isotropic linear elastic equations for each layer have the following general solution in terms of spherical coordinates $r, \theta, \phi$ (see [28]):

$$
\begin{aligned}
u_{r} & =\alpha r+\frac{\beta}{r^{2}}, \\
\epsilon_{r r} & =\alpha-2 \frac{\beta}{r^{3}}, \quad \epsilon_{\theta \theta}=\epsilon_{\phi \phi}=\alpha+\frac{\beta}{r^{3}}, \\
\sigma_{r r} & =3 \kappa \alpha-4 \mu \frac{\beta}{r^{3}}, \quad \sigma_{\theta \theta}=\sigma_{\phi \phi}=3 \kappa \alpha+2 \mu \frac{\beta}{r^{3}},
\end{aligned}
$$

where $\alpha$ and $\beta$ are constants to be determined. The solutions (23)-(24) are given within each shell for appropriate values of the elastic moduli and different values of the constants $\alpha, \beta$.

Two different cases of composites are studied. In the first case (a), note that the contact between the two shells is modeled as perfect, and in the second case (b), an elastic intermediate interphase is inserted between the two shells with perfect contact.

In the following, the cases (a) and (b) are solved using a SAM. The model proposed by SAM has been obtained in [13] as an asymptotic model of a thin curved elastic adhesive. 


\section{I. Case (a): perfect contact between the inner and the outer shells}

Under perfect contact conditions between the two shells, the radial stress and displacement are continuous at the common interface of radius $R_{0}:=R_{i}+t=R_{e}-t$. Using equations (22) and (24), the following set of boundary (25) and (28) and contact (26) and (27) conditions is obtained:

$$
\begin{array}{r}
3 \kappa_{i} \alpha_{i}-4 \mu_{i} \frac{\beta_{i}}{R_{i}^{3}}=-p, \\
3 \kappa_{i} \alpha_{i}-4 \mu_{i} \frac{\beta_{i}}{R_{0}^{3}}=3 \kappa_{e} \alpha_{e}-4 \mu_{e} \frac{\beta_{e}}{R_{0}^{3}}, \\
\alpha_{i} R_{0}+\frac{\beta_{i}}{R_{0}^{2}}=\alpha_{e} R_{0}+\frac{\beta_{e}}{R_{0}^{2}}, \\
3 \kappa_{e} \alpha_{e}-4 \mu_{e} \frac{\beta_{e}}{R_{e}^{3}}=0 .
\end{array}
$$

These conditions uniquely determine $\alpha_{i}, \beta_{i}$ for the inner shell and $\alpha_{e}, \beta_{e}$ for the outer shell. Given these four constants, the relations (22) and (24) provide the displacement and stress fields in each shell.

\subsection{Case (b): contact via an elastic layer between the inner and the outer shells}

It is now assumed that the contact between the inner and the external shell is provided by the presence of a thin elastic interphase of radius $R_{0}, R_{i}<R_{0}<R_{e}$ and small thickness $\varepsilon \gg t$, inserted between the two shells. The interphase is in perfect contact with the two adjacent shells at the common surfaces $r=R_{0} \pm \varepsilon$ and each shell is made of a linear isotropic material with elastic moduli $\mu, k$, see Figure 1.

Taking into account the general relations (22) and (24), the following set of boundary (29) and (34) and contact (30)-(33) conditions is now obtained

$$
\begin{array}{r}
3 \kappa_{i} \alpha_{i}-4 \mu_{i} \frac{\beta_{i}}{R_{i}^{3}}=-p, \\
3 \kappa_{i} \alpha_{i}-4 \mu_{i} \frac{\beta_{i}}{\left(R_{0}-\varepsilon\right)^{3}}=3 \kappa \alpha-4 \mu \frac{\beta}{\left(R_{0}-\varepsilon\right)^{3}}, \\
3 \kappa \alpha-4 \mu \frac{\beta}{\left(R_{0}+\varepsilon\right)^{3}}=3 \kappa_{e} \alpha_{e}-4 \mu_{e} \frac{\beta_{e}}{\left(R_{0}+\varepsilon\right)^{3}}, \\
\alpha_{i}\left(R_{0}-\varepsilon\right)+\frac{\beta_{i}}{\left(R_{0}-\varepsilon\right)^{2}}=\alpha\left(R_{0}-\varepsilon\right)+\frac{\beta}{\left(R_{0}-\varepsilon\right)^{2}}, \\
\alpha\left(R_{0}+\varepsilon\right)+\frac{\beta}{\left(R_{0}+\varepsilon\right)^{2}}=\alpha_{e}\left(R_{0}+\varepsilon\right)+\frac{\beta_{e}}{\left(R_{0}+\varepsilon\right)^{2}}, \\
3 \kappa_{e} \alpha_{e}-4 \mu_{e} \frac{\beta_{e}}{R_{e}^{3}}=0 .
\end{array}
$$

These conditions uniquely determine $\alpha_{i}, \beta_{i}$ for the inner shell, $\alpha, \beta$ for the interphase and $\alpha_{e}, \beta_{e}$ for the outer shell. Given these six constants, the relations (22) and (24) again provide the displacement and stress fields inside the two shells and the interphase.

\section{Comparison of the numericals results}

In the present section, the numerical results obtained via AHM and SAM for the two cases (a) and (b) of the pressurized spherical shells assemblage presented in the above section are compared. The comparison is given in terms of radial displacement, radial and angular stresses. The values of the material parameters used in the 
Table I. Comparison of the normalized radial stress $\sigma_{r r}$ for the points $R_{i}, R_{0}$ and $R_{e}$ computed using AHM and SAM for two, four, eight and an infinite number of layers with perfect contact (case (a)).

\begin{tabular}{lccc}
\hline Methods & \multicolumn{3}{c}{$\sigma_{r r}(\cdot) / p$} \\
\cline { 2 - 3 } & $R_{i}$ & $R_{0}$ & $R_{e}$ \\
\hline SAM(2 layers) & -1 & -0.08964 & 0 \\
SAM(4 layers) & -1 & -0.3948 & 0 \\
SAM(8 layers) & -1 & -0.3953 & 0 \\
SAM $+\infty$ & -1 & -0.3969 & 0 \\
AHM & -1 & -0.3959 & 0 \\
\hline
\end{tabular}

calculations are taken as the following:

$$
\begin{aligned}
& \mu_{i}=10 \mu_{e}, \quad \mu=10^{x} \mu_{e}, \\
& v_{i}=0.2, \quad v_{e}=0.35, \quad v=0.3, \\
& t=R_{0} / 10, \quad \varepsilon=R_{0} / 100,
\end{aligned}
$$

where the non-indexed constants are the interphase elastic parameters of the models (b) described in previous subsections. So, the elastic properties for case (a) are constant for the variable $x$.

The variable $x=\log _{10}\left(\mu / \mu_{e}\right)$ is introduced to model different stiffness ratios between the material of the adhesive interphase and the material of the external shell. In particular, for a fixed Lamé constant of the external shell $\mu_{e}$, the adhesive interphase becomes softer as $\mu \rightarrow 0$ and correspondingly $x \rightarrow-\infty$; conversely, the limit $x \rightarrow+\infty$ corresponds to a rigid adhesive.

The numerical results of the displacement via AHM are computed using the solution of the equation (19) considering the homogenized coefficients from equation (15). The circumferential and radial stresses are computed using the equations (16) and (18), respectively, considering the effective coefficients of equation (15).

The two models presented in the previous section, under pressurized SAM, the radial displacement and the radial and angular stresses are computed solving the two system sets of boundary and contact conditions (25)-(28) and (29)-(34).

\section{I. Numerical results for case (a)}

A spherical shell composite model is considered with thickness $2 t$. Two layers, one external " $e$ " and one internal " $i$ " have been distributed periodically inside the thickness of this composite. In addition a layer sequence is constructed considering an assemblage with more layers as follows:

$$
\begin{aligned}
& 2 \text { layers: } i / e \\
& 4 \text { layers: } i / e / i / e \\
& 8 \text { layers: } i / e / i / e / i / e / i / e
\end{aligned}
$$

The numerical results obtained via AHM are based on the solution (20) with boundary conditions given by (21) in the inner surface and free traction in the external surface. The results are compared using SAM for the above-mentioned distribution of layers sequences and infinite number of layers.

In Table 1, the normalized radial stress obtained via SAM for two, four and eight layers is compared with AHM using (18). The case of infinite numbers of layers computed by SAM has been computed considering the results obtained in [29, Equations (51)]. Note the good agreement between the two proposed methods.

The circumferential stress in a thin spherical shell under internal pressure is given by the well-known estimation

$$
\sigma_{\theta \theta}=\frac{2 p r}{4 T}
$$

where $r$ is the radius and $T=2 t$ is the thickness of the composite [30]. Based on the equilibrium considerations [31, Chap. 9], the estimation (38) can be applied to the spherical composite assemblage considered in this work. It has to be remarked that equation (38) provides an approximate value of the hoop stress, because (38) neglects the elastic behavior of the shells constituting the composite and the contact between them. On the other hand, 
Table 2. Comparison of the normalized angular stress $\sigma_{\theta \theta}$ for the points $R_{i}, R_{0}$ and $R_{e}$ computed using AHM, SAM for two, four, eight and an infinite number of layers and hoop stress formula (38) with perfect contact (case (a)).

\begin{tabular}{llll}
\hline Methods & & $\sigma_{\theta \theta}(\cdot) / p$ & $R_{e}$ \\
\cline { 2 - 3 } & $R_{i}$ & $R_{0}$ & 0.4062 \\
SAM(2 layers) & 4.0389 & $0.4510(\mathrm{e})$ & 0.4177 \\
SAM(4 layers) & & $3.4955(\mathrm{i})$ & $0.2850(\mathrm{e})$ \\
SAM(8 layers) & 4.3070 & $3.5916(\mathrm{i})$ & 0.4255 \\
SAM $+\infty$ & & $0.2981(\mathrm{e})$ & 1.7935 \\
AHM & 4.4697 & 2.0091 & 1.7875 \\
Hoop stress & 2.3794 & 2.0071 & 2.2500 \\
\hline
\end{tabular}

Table 3. Comparison of the normalized displacement $u_{r}$ for the points $R_{i}, R_{0}$ and $R_{e}$ computed using AHM and SAM for two, four, eight and an infinite number of layers with perfect contact (case (a)).

\begin{tabular}{llcr}
\hline Methods & \multicolumn{3}{c}{$\mu_{e} u_{r}(\cdot) /\left(p R_{0}\right)$} \\
\cline { 2 - 4 } & $R_{i}$ & $R_{0}$ & $R_{e}$ \\
\hline SAM(2 layers) & 0.1287 & 0.1202 & 0.1076 \\
SAM(4 layers) & 0.1367 & 0.1198 & 0.1106 \\
SAM(8 layers) & 0.1416 & 0.1230 & 0.1127 \\
SAM $+\infty$ & 0.1641 & 0.1423 & 0.1297 \\
AHM & 0.1470 & 0.1267 & 0.1151 \\
\hline
\end{tabular}

estimates of this type are widely used by engineers and thus it is worth comparing with the results given by equations (16) and (24).

In Table 2, a comparison of the normalized $\sigma_{\theta \theta}$ computed via AHM, SAM and the hoop stress using equation (38) is shown. The stress $\sigma_{\theta \theta}$ is computed via AHM and SAM using equations (16) and (24), respectively, for two, four, eight and an infinite number of layers.

The results obtained using SAM show a discontinuity of $\sigma_{\theta \theta}$ in $R_{0}$ for two, four and eight layers, see Table 2. Otherwise, the case of infinite number of layers SAM $+\infty$ is continuous because it corresponds to the case of a homogenized material. A good coincidence between the results obtained by AHM and SAM $+\infty$ is appreciated.

In Table 3, the normalized displacements $u_{r}$ obtained using AHM and SAM for two, four, eight and infinite number of layers are shown. The displacement function is computed via AHM using the equation (20) for a transversely isotropic structure. The values of $u_{r}$ are obtained via SAM using the equation (23) and the solution of the system (25)-(28). A good agreement between the two methods is observed.

The average behavior of the $\sigma_{\theta \theta}$ is studied due to the discontinuity of the function at the interfaces points (see Table 2, SAM), the following operator is introduced to computed the average of the numerical curves

$$
\hat{\varphi}=\frac{1}{2}\left(\varphi\left(R_{i}\right)+\varphi\left(R_{e}\right)\right)
$$

where $\varphi$ is taken to denote the displacement $u_{r}$, the radial stress $\sigma_{r r}$ or the angular stress $\sigma_{\theta \theta}$.

In Table 4, the average normalized stresses and radial displacement computed for the above sequences via SAM and AHM are reported. Note that the average normalized radial displacement augments as the number of layers increases and the value computed by SAM $+\infty$ provides an upper bound, as is expected. This is not the case of the angular stress but this discrepancy is believed to appear due to the choice of average (39) which is not able to capture the oscillating behavior of the angular stress inside the thickness of the composite as the number of layers increases. Indeed, the mean value given by the following expression is introduced

$$
\bar{\varphi}=\frac{1}{R_{0}^{2}} \int_{R_{i}}^{R_{e}} \varphi(r) r d r .
$$


Table 4. Comparison of average (according to Equation (39)) normalized stresses and radial displacement in a hollow composite assemblage with an increasing number $n$ of layers with perfect contact. The inner surface of the composite is subject to the pressure $p$, equation (2I), the external surface of the composite is free traction. The values for case SAM $+\infty$ have been computed by using equation (5I) of [29].

\begin{tabular}{lccc}
\hline Methods & $\hat{\sigma}_{r r} / p$ & $\hat{\sigma}_{\theta \theta} / p$ & $\mu_{e} \hat{u}_{r} /\left(p R_{0}\right)$ \\
\hline SAM(2 layers) & -0.5 & 2.2226 & 0.1182 \\
SAM(4 layers) & -0.5 & 2.3624 & 0.1237 \\
SAM(8 layers) & -0.5 & 2.4476 & 0.1271 \\
SAM $+\infty$ & -0.5 & 2.0865 & 0.1469 \\
AHM & -0.5 & 2.0916 & 0.1311 \\
\hline
\end{tabular}

Table 5. Comparison of average (according to equation (40)) normalized stresses and radial displacement in an hollow composite assemblage for an increasing number $n$ of layers with perfect contact. The inner surface of the composite is subject to the pressure $p$ (cf. equation (2I)), the external surface of the composite is free traction. The values for case SAM $+\infty$ have been computed by using Equations (5I) of [29].

\begin{tabular}{lccc}
\hline$n$ & $\bar{\sigma}_{r r} / p$ & $\bar{\sigma}_{\theta \theta} / p$ & $\mu_{e} \bar{u} /\left(p R_{0}\right)$ \\
\hline SAM(2 layers) & -0.0523 & 0.4050 & 0.0237 \\
SAM(4 layers) & -0.0664 & 0.4050 & 0.0245 \\
SAM(8 layers) & -0.0743 & 0.4050 & 0.0250 \\
SAM $+\infty$ & -0.0829 & 0.4050 & 0.0287 \\
AHM & -0.0828 & 0.4050 & 0.0255 \\
\hline
\end{tabular}

The values listed in Table 5 are obtained using the mean value (40). Table 5 shows that all of the average (according to (40)) normalized values estimated for SAM $+\infty$ provide upper or lower (depending on the sign) bounds for the values computed by SAM with two, four and eight layers. The good coincidence between the results obtained via AHM and SAM $+\infty$ is noticeable.

\subsection{Numerical results for case (b)}

A spherical shell composite with thickness $2 t$ is considered in which two layers " $i$ " and " $e$ " have a thin interphase between the laminates. The layers and the interphase with perfect contact are distributed periodically inside the thickness and along the radial axis. The structure is studied with boundary conditions given by (21) in the inner surface and free traction in the external surface.

In order to compare the results obtained via AHM and SAM, the angular and radial stresses are computed at the points $R_{i}, R_{0}, R_{e}$ of the spherical structure for different number of layers. The sequences of the layers used to calculate the stresses and the displacements via SAM have the following distribution of the layers inside the thickness

$$
\begin{aligned}
& 2 \text { layers: } i \text { /TI /e } \\
& 4 \text { layers: } i \text { /TI /e / } i \text { /TI /e } \\
& 8 \text { layers: } i \text { /TI /e / } i \text { /TI /e / } / \text { TI /e / } i \text { /TI /e }
\end{aligned}
$$

where (TI) is the thin interphase between the layers " $i$ " and " $e$ ".

The numerical results are computed for the values $x=\log \left(\mu / \mu_{e}\right) \in[-3,3]$ and the elastic parameters given in (35)-(36). As with the above case (case (a)), the numerical results for case (b) obtained via AHM are based on the solution (20). The functions computed via SAM are derived from the solution of the system (29)-(34) considering the aforementioned distribution for two, four and eight layers. The case of an infinite number of layers computed by SAM is studied considering the results reported in [29, Equations (51)].

Figure 2 shows the behavior of the normalized radial stress $\sigma_{r r}$ at $R_{0}$ for different values of the parameter $x$. The computation of $\sigma_{r r}$ via AHM is computed using the equation (18). The results are compared with the values obtained via SAM for two, four, eight and an infinite number of layers, using the corresponding equation (24). A good agreement between the curves is appreciated, except for SAM two layers. This is due to the microstructure 


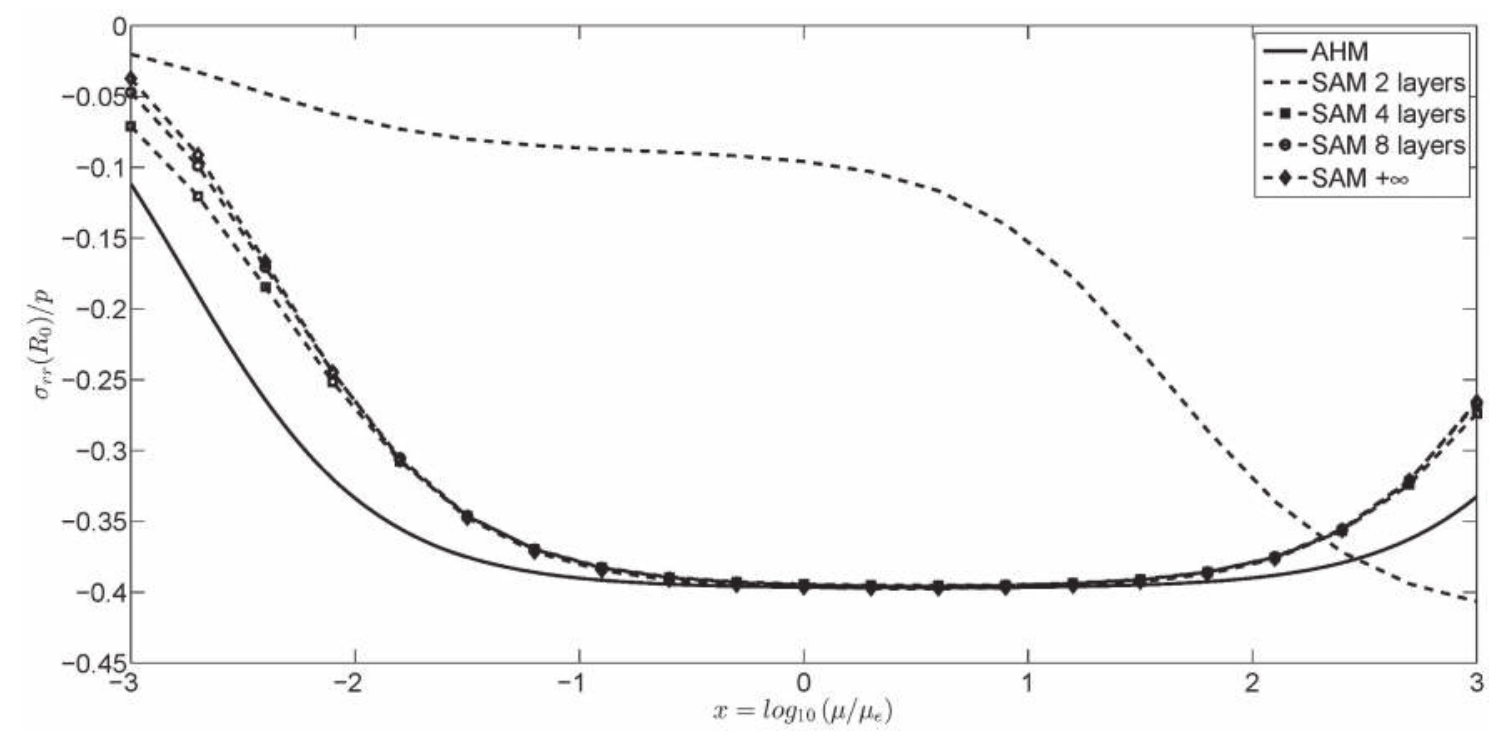

Figure 2. Normalized radial stress $\sigma_{r r}$ at $R_{0}$ for case (b) using AHM and SAM with two, four, eight and an infinite number of layers.

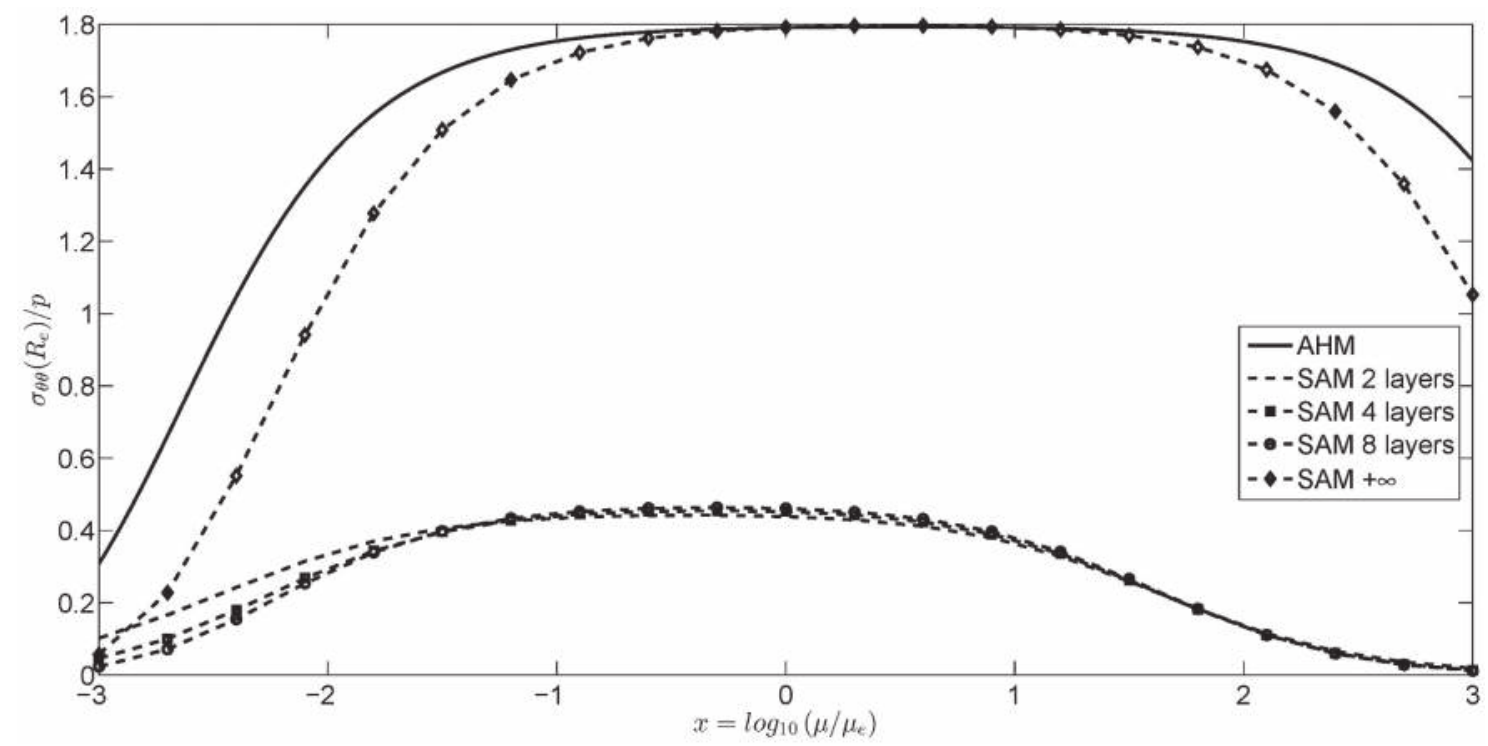

Figure 3. Normalized angular stress $\sigma_{\theta \theta}$ at $R_{e}$ for case (b) using AHM and SAM with two, four, eight and an infinite number of layers.

above described, the adhesive interphase (TI) lies at $r=R_{0}$ (the middle surface of the composite) for SAM two layers, whereas for more layers than two, the adhesive interphase does not lie on $R_{0}$.

In Figure 3, a comparison between the normalized $\sigma_{\theta \theta}$ at $R_{e}$ computed via AHM and SAM for two, four, eight and an infinite number of layers is shown. The computation via SAM are obtained from the corresponding equation (24). The values of $\sigma_{\theta \theta}$ computed using AHM is given by equation (16). A good agreement of the behavior of the curves obtained via AHM and SAM for infinite number of layer is appreciated.

Figure 4 shows the behavior of the normalized displacement $u_{r}$ at $R_{i}$. The curves are determined using AHM and SAM for two, four, eight and an infinite number of layers following a similar idea: the displacement function is computed via AHM using equation (20) and via SAM (23).

A good coincidence between both methods can be observed, especially with the results obtained by AHM and SAM $+\infty$ show a perfect match for the values of the parameter $x \in[-1,2]$. The differences between $\mathrm{AHM}$ and SAM $+\infty$ for $x$ out of $[-1,2]$ is related to the convergence difficulties of the AHM for high-contrast 


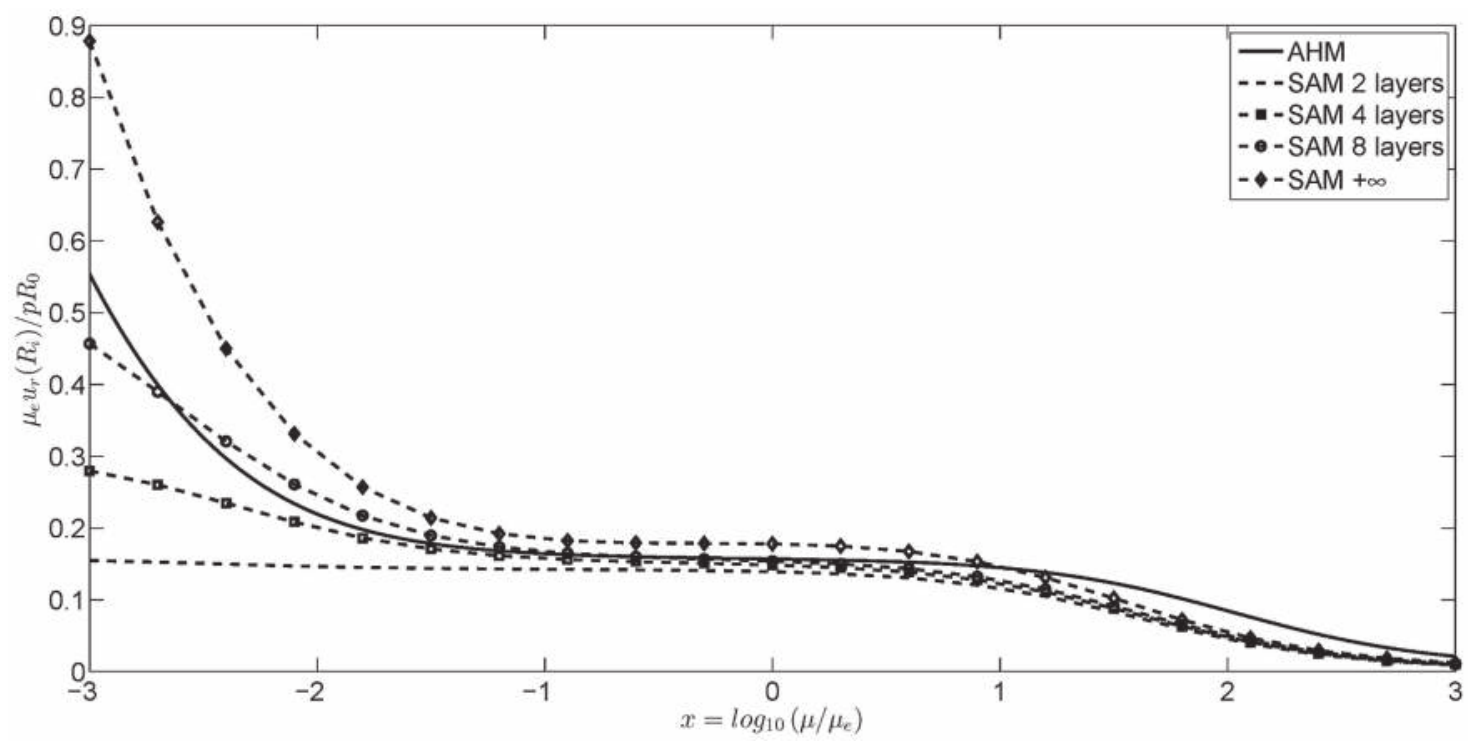

Figure 4. Normalized displacement $u_{r}$ at $R_{i}$ for case (b) using AHM and SAM with two, four, eight and an infinite number of layers.

composite $[32,33]$. Finally, if the thickness of the interphase layer is considered very small, then the curves given in Figures 2, 3 and 4 show a constant behavior given in Tables 1, 2 and 3, respectively.

\section{Conclusions}

In this work, the equilibrium problem of a multi-layered laminated spherical composite has been solved using the two-scale AHM and SAM. The numerical computations were made considering two different conditions of contact at the interface of the layers: (a) perfect contact condition and (b) elastic interphase layer between the inner and the outer shells. During the homogenization process, the global behavior of a shell composite made of isotropic layers has transversely isotropic symmetry. The solution of the homogeneous problem for a transversely isotropic elastic spherical structure has been obtained. The results of the displacement, radial and circumferential stress obtained by AHM have been compared with the same obtained fields using the method proposed in [13] (SAM). Both methods show good agreement between their solution and with the experimental estimate reported in [30]. The computational cost for a great number of layers is not a problem for the AHM model. This results opens the possibility to extend the computation to composites made of a large number of layers, with the aim to apply the computation and the model in a particular group of materials and bio-composites, veins, arteries and cornea. The study of the elastic properties of the cornea and veins is an important objective in medicine today. Considering this idea the formulation of a mathematical model of the cornea and aorta will be addressed in a future work by taking into account composites exhibiting different types of geometries and imperfect contact conditions at the interfaces.

\section{Acknowledgments}

Thanks are due to Professor Francesco del Isola for his fruitful comments. Thanks also go to Departamento de Matemáticas y Mecánica, IIMAS-UNAM, for its support and Ramiro Chávez Tovar and Ana Pérez Arteaga for computational assistance. SHIHMAS Interfaces rigides et souples dans les matriaux et structures hétérogènes (Soft and hard interfaces for heterogeneous materials and structures), 2016 sponsored by French Embassy in La Habana is also grateful.

\section{Funding}

The author(s) disclosed receipt of the following financial support for the research, authorship, and/or publication of this article: This work was supported by by FSP (Cooperation Scientifique Franco-Cubaine) Cuba 2011-26 (project SHICHAN number 29935XH) and the University of Havana (project Composite Materials). 


\section{References}

[1] Reddy, J. Mechanics of laminated composite plates and shells. Theory and analysis. Boca Raton, FL: CRC Press, 2004.

[2] Strek, T, Jopek, H, Maruszewski, B, et al. Computational analysis of sandwich- structured composites with an auxetic phase. Physics Status Solidi B 2014; 251: 354-366.

[3] Fessel G, Broughton J, Fellows $\mathrm{N}$ et al. Evaluation of different lap-shear joint geometries for automotive applications. Int $J$ Adhesion Adhesives 2007; 27: 574-583.

[4] Sapuan, S, Maleque, MA, Hameedullah, M, et al. A note on the conceptual design of polymeric composite automotive bumper system. J Mater Process Technol 2005; 159: 145-151.

[5] Fillep, S, Mergheim, P, and Steinmann, P. Computational modelling and homogenization of technical textiles. Eng Struct 2013; 50: 68-73.

[6] Peng, X, and Cao, J. A dual homogenization and finite element approach for material characterization of textile composites. Composite B 2002; 33: 45-56.

[7] Murakami, M, Kondoh, M, Iwai, Y, et al. Measurement of aerodynamic forces and flow field of a soccer ball in a wind tunnel for knuckle effect. Procedia Engineering 2010; 2: 2467-2472.

[8] Cross, R. Physics of Baseball and Softball. New York: Springer, 2011.

[9] Nathan, A. The effect of spin on the flight of a baseball. Am Assoc Phys Teachers 2008; 76: 119-124.

[10] Hashin, Z. Thin interphase imperfect interface in elasticity with application to coated fiber composites. J Mech Physics Solids 2002; 50: 2509-2537.

[11] Vasilenko, A, Emel'yanov, I and Kuznetsov, V. Stress analysis of laminated shells of revolution with an imperfect interlayer contact. Int Appl Mech 2001; 37: 662-669.

[12] Aboudi, J. Damage in composites-modeling of imperfect bonding. Composites Sci Technol 1987; 28: 103-128.

[13] Rizzoni, R, and Lebon, F. Imperfect interfaces as asymptotic models of thin curved elastic adhesive interphase. Mech Res Commun 2013; 51: 39-50.

[14] Chen, YC, Rajagopal, KR, and Wheeler, L. Homogenization and global responses of inhomogeneous spherical nonlinear elastic shells. J Elasticity 2006; 82: 193-214.

[15] Lutoborski, A. Homogenization of linear elastic shells. J Elasticity 1985; 15: 69-87.

[16] Saha, G, Kalamkarov, AL, and Georgiades, AV. Asymptotic homogenization modeling and analysis of the effective properties of smart composite reinforced and sandwich shells. Int J Mech Sci 2007; 49: 138-150.

[17] Ding, H, Chen, W, and Liangchi, Z. Solid Mechanics and its applications: Elasticity of Transversely Isotropic materials. Dordrecht: Springer, 2006.

[18] Frydmann, M. Determinations of the dynamics elastic constants of a transverse isotropic rock based on borehole dipole sonic anisotropy in deviated wells. In Rio Oil and Gas Expo and Conference 2010 Proceedings, vol. 1, pp. 1-10.

[19] Weiss, JA, Maker, BN, and Govindjee, S. Finite element implementation of incompressible, transversely isotropic hyperelasticity. Comput Meth Appl Mech Eng 1996; 135: 107-128.

[20] Yoon, YJ, Yang, G, and Cowin, C. Estimation of the effective transversely isotropic elastic constants of a material from known values of the material's orthotropic elastic constants. Biomech Model Mechanobiol 2002; 1: 83-93.

[21] Pobedrya, B. Mechanics of Composite Materials. Moscow: Moscow State University Press, 1984.

[22] Tsalis, D, Chatzigeorgiou, G, and Charalambakis, N. Homogenization of structures with generalized periodicity. Composites B 2012; 43: 2495-2512.

[23] Ciarlet, PG. Mathematical Elasticity: Theory of shells, vol. III. Amsterdam: Elsevier Science B. V., 2000.

[24] Lavrentyev, I, and Rokhlin, S. Ultrasonic spectroscopy of imperfect contact interfaces between a layer and two solids. $J$ Acoust Soc Am 1998; 103: 657-664.

[25] Bakhvalov, N, and Panasenko, G. Homogenisation: Averaging Processes in Periodic Media. Moscow: Mathematics and its Application (Soviet Series), 1989.

[26] Cioranescu, D, and Donato, P. An Introduction to Homogenization. New York: Oxford University Press, 1999.

[27] Guinovart-Sanjuán, D, Rodríguez-Ramos, R, Guinovart-Díaz, R, et al. Effective properties of regular elastic laminated shell composite. Composites B 2016; 87: 12-20.

[28] Love, A. A Treatise on the Mathematical Theory of Elasticity. New York: Dover Publications, 1944.

[29] Bufler, H. The arbitrarily and the periodically laminated elastic hollow sphere: exact solutions and homogenization. Arch Appl Mech 1998; 68: 579-588.

[30] Ibrahim, A, Ryu, Y, and Saidpour, M. Stress analysis of thin-walled pressure vessels. Modern Mech Eng 2015; 5: 1-9.

[31] Hearn, E. Mechanics of Materials 1. London: Butterworth Heinemann, 2000.

[32] Briane, M, and Canar-Eddine, M. Homogenization of two-dimensional elasticity problems with very stiff coefficients. $J$ Math Pures Appl 2007; 88(6): 483-505.

[33] Adrianov, I, Bolshkov, V, Danishevs'kyy, V, et al. Higher order asymptotic homogenization and wave propagation in periodic composite materials. Proc R Soc A 2008; 464: 1181-1201. 